The development of German children's moral reasoning about prosocial conflicts, and the interrelations of moral reasoning and prosocial behavior were examined. Hedonistic reasoning decreased in usage from the preschool years to fourth grade; direct reciprocity, needs-oriented, approval/interpersonal, and reasoning involving references to others' humanness increased with age. The pattern of development was strikingly similar to that of an American sample of the same age. However, German children used more direct reciprocity and less stereotypic reasoning than did American children. As has been found for American children, sharing behavior but not helping was related to level of moral judgment. The results are discussed in relation to theory and the existing research concerning prosocial development in America.

\title{
THE DEVELOPMENT OF PROSOCIAL BEHAVIOR AND COGNITIONS IN GERMAN CHILDREN
}

\author{
NANCY EISENBERG \\ Arizona State University \\ KLAUS BOEHNKE \\ PETRA SCHUHLER \\ RAINER K. SILBEREISEN \\ Technische Universitaet Berlin
}

In the past fifteen years, there has been considerable research related to issues such as developmental changes in cognitions concerning altruism and the relation of these cognitions to prosocial behavior (e.g., Underwood \& Moore, 1982). Nonetheless, there are clear limitations to conclusions that can be drawn from existing data. This is due, in part, to inconsistencies in research findings and to the fact that most of the existing

\footnotetext{
AUTHORS' NOTE: This research was supported by grants from the Deutscher Akademischer Austauschdienst (German Academic Exchange Service) and the Provost Fund at Arizona State University to the first author, and by the Psychology Department at the Technische Universitaet in
} 
research has been conducted with American middle-class children.

The study of children's reasoning about the resolution of moral conflicts involving prosocial behavior has been an outgrowth of Kohlberg's (1969) research on moral judgment. Kohlberg has assumed that his stages of moral judgment are universal across cultures, and apply to the entire range of moral issues. However, Kohlberg's moral dilemmas generally de-emphasize issues related to caring and prosocial behavior and emphasize prohibition-oriented issues, such as issues relating to laws, authorities and their dictates, rules, formal obligations, and punishment. Children's reasoning about prosocial dilemmas - those in which the needs or wants of one individual conflict with those of another in a situation in which the role of laws, rules, punishments, authorities, and formal obligations is minimal (Eisenberg-Berg, 1979; O'Connor, Cuevas, \& Dollinger, 1981) - appears to differ somewhat from that delineated in Kohlberg's stages.

More specifically, with regard to prosocial moral judgment, researchers have found that young children verbalize relatively little reasoning concerning blind deference to authority or fear of punishment. Rather, children use much hedonistic and needs-oriented (primitive empathic) reasoning. In elementary school, children's judgments have been found to reflect, to some degree, approval-oriented considerations, and the desire to behave in stereotypically "good" ways (as well as needsoriented and hedonistic reasoning). By high school age, children frequently verbalize self-reflective empathic reasoning, or reasoning concerning internalized abstract principles and internalized affective reactions (e.g., guilt or positive affect relating to living up to one's principles; see Eisenberg-Berg, 1979; O'Connor et al., 1981). Although the pattern of develop-

Berlin. The German authors are presented in alphabetical order. We would like to express our gratitude to the following persons for assisting in the data collection: Annette Claar, Randy Lennon, Susanne Nagel, Jeannette Pasternack, Karlsson Roth, Juergen Schreckling, Wolfgang Spellbrink, and Wayne Stutzer. Requests for reprints should be addressed to Nancy Eisenberg, Department of Psychology, Arizona State University, Tempe, AZ 85287. 
ment described above has been found in both cross-sectional and longitudinal research involving American children, it was unclear whether children in other cultures would exhibit a similar pattern of development. Thus, one of the primary purposes of the present research was to examine the development of children's reasoning about prosocial moral conflicts in another country, Germany.

The developmental changes in prosocial moral judgment, like those delineated by Kohlberg (1969), are believed to be a function, in part, of advances in cognitive role-taking skills and the capacity for logical thought. Consequently, one would predict considerable similarity in the development of prosocial moral judgment across cultures. However, experiential factors also seem to contribute to the observed pattern of development. Thus, one would also predict that there might be some differences in the prosocial reasoning of children from different countries. For example, although Adelson (1971) found that German children were more likely than American or English children to trust authorities, view authorities as wise and benevolent, and to advocate strong leadership, as of 1978 American adolescents were found to score somewhat higher on authoritarianism (Lederer, 1982). Consequently, it is possible that German and American children will differ in the use of a variety of types of moral judgment (e.g., authority-oriented, approval/interpersonal, or internalized law or norm reasoning).

Individual differences in level of moral judgment seem to be a consequence, in part, of variations in socialization histories. In specific, researchers have found that the moral judgment levels of Kohlberg (e.g., Leahy, 1979; Parikh, 1980) and prosocial (e.g., Eisenberg, Lennon, \& Roth, 1983) reasoning are positively related to nonrestrictive childrearing practices and, to some degree, maternal warmth. Thus, cross-national differences in childrearing practices are likely to be associated with cross-national variations in moral judgment. According to children's reports, German parents appear to provide more love and support than do American parents (Devereux, 
Bronfenbrenner, \& Suci, 1962). Given the association between maternal warmth and prosocial moral judgment in the early but not mid-elementary school years (Eisenberg et al., 1983), one might predict that young German children's prosocial moral judgment would be more advanced than that of their American counterparts. However, the fact that American mothers apparently allow their 6 and 14 year-old children more autonomy than do German mothers (Wesley \&Karr, 1968) is consistent with the hypothesis that American children may be more advanced in reasoning in the later school years.

Although research on this issue is scarce, the relation between prosocial cognitions and performance of prosocial behavior seems, in general, to be positive (Underwood \& Moore, 1982). Moreover, in research with American children it appears that mode of prosocial moral judgment (especially reasoning reflecting self-oriented or other-oriented concerns) is more likely to be positively associated with costly prosocial actions such as sharing or donating than with simple acts of helping that are low in cost, take little time, and do not require the forfeiture of an object (Eisenberg, Pasternack, \& Lennon, 1984; Eisenberg-Berg \& Hand, 1979). However, few researchers have investigated the relation of prosocial reasoning to more than one type of prosocial behavior, or have examined this relation with children other than Americans. Thus, the final purpose of the present study was to further examine the relation between prosocial actions and reasoning.

\section{METHOD}

\section{SUBJECTS}

The German participants were 30 preschoolers ( 16 girls, 14 boys), 29 second graders (13 girls, 16 boys), and 33 fourth graders ( 17 girls, 16 boys) from West Berlin. The children were nearly all Caucasian, and from working and middle-class families. The mean ages (in months) for the preschoolers, 
second graders, and fourth graders were 62 (range $=60-72), 94$ (range $=86-110)$, and 117 (range $=108-134)$, respectively.

The American children were 30 preschoolers (16 girls, 14 boys), 30 second graders (12 girls, 18 boys) and 39 fourth graders ( 22 girls, 17 boys). All except nine were Caucasian. The mean ages of the preschoolers, second, and fourth graders were 63 (range $=57-68), 95$ (range $=88-109)$, and 117 (range $=$ 108-130) months, respectively.

\section{INSTRUMENTS}

Prosocial moral judgment instrument. Prosocial moral judgment was assessed with four illustrated moral dilemmas (Eisenberg-Berg \& Hand, 1979). Each dilemma depicted a situation in which the needs and wants of the story protagonist were in conflict with those of another (or others) in a situation in which the role of authorities, punishment, laws, rules, and formal obligations was minimal. The stories were slightly modified for the German children, such that the word "courtyard" was used instead of "yard."

Prosocial behavior. The older German children were given the opportunity to donate anonymously (8 candies) to poor orphans. Helping behavior was also assessed by noting the number of fallen paper clips picked up by the child in 60 seconds (the experimenter accidently dropped 75 clips and picked up 30 clips slowly).

\section{PROCEDURES}

The German elementary school children were interviewed individually by two males and two females; the preschoolers by a male and a female. For the elementary school children, the interview began with the paper clips helping task. Next, the prosocial dilemmas were presented orally in random order. Standardized probes were used, and the children retold each story to check for understanding. Then the children were given 8 candies for participation and were left alone for two minutes, during which time they could donate to poor orphans. The 
German preschoolers were administered only the moral judgment stories.

The American children were interviewed by three females and one male. They were administered only the moral dilemmas.

\section{SCORING}

The primary coder for all the moral judgment protocols was an American who had translated versions of the German protocols. The two reliability coders for the German data were German researchers who spoke English (means of the Germans' codings were used for reliability), whereas the reliability coder for the American data was an American. Scoring was done in two ways. First, the children's moral judgments were coded into categories derived from Eisenberg-Berg (1979) as follows; only those used with some frequency are defined.

Hedonistic reasoning: (1) pragmatic, hedonistic gain to the selforientation to selfish gain for oneself (in addition to gain resulting from direct reciprocity); (2) direct reciprocityorientation to personal gain due to direct reciprocity (or lack of it) from the recipient of an act; (3) affectional relationship - the individual's identification with another, his or her liking for the other, and the other's relation to his or her own needs are important considerations in the individual's moral reasoning.

Nonhedonistic pragmatism: orientation to practical concerns that are unrelated to selfish considerations.

Needs-oriented reasoning: orientation to the physical, material, or psychological needs of the other person when the other's needs conflict with those of the story protagonist, such as "He needs blood."

Stereotypes of a good or bad person: orientation to stereotyped images of a good or bad person or behaviors.

Approval and interpersonal orientation: orientation to others' approval and acceptance in deciding what is the correct behavior.

Self-reflective empathic orientation: (1) sympathetic orientationexpression of sympathetic concern and caring for others; (2) 
role-taking - the individual takes the perspective of the other and explicitly uses this perspective in his or her reasoning; (3) reference to and concern with humanness-orientation to the fact that the other is human, living, a person.

Internalized affect: simple internalized positive affect and positive affect related to consequences. The individual states that he or she would feel good as a result of a particular course of action, either without giving a reason or due to the consequences of his or her act for the other person.

Each child was assigned scores indicating the frequency that he or she used each type of reasoning when discussing both the pros and cons of helping the needy other $(1=$ no use of category; 4 = a major type of reasoning used). Next, the scores for each category were summed across the four stories (potential range $=4-16$ ). Interrater reliabilities for the German and American subjects computed for each of the reasoning categories used in the analyses ranged from .77 to 1.00 (all frequently used ones were above .80 ) and .75 to 1.00 , respectively, with the exception of several advanced categories that were scored once by the primary coder and never by the reliability coders (and were not used in analyses).

The above categories of reasoning are viewed as representing components of developmental levels of prosocial moral judgment. In prior studies, these categories were grouped into levels on the basis of factor analyses, linear trend analyses (used to determine age changes in reasoning), and other analyses of age-related change (see Eisenberg et al., 1983; Eisenberg-Berg, 1979). The resultant levels are:

Level 1: Hedonistic, self-focused orientation-reasons for assisting or not assisting another include consideration of direct gain to the self, future reciprocity, and concern for others whom the individual needs and / or likes (due to the affectional tie).

Level 2: "Needs of others" orientation-individual expresses concern for the physical, material, and psychological needs of others even though the other's needs conflict with one's own needs. This concern is expressed in the simplest terms, without clear evidence of self-reflective role taking or verbal expressions of sympathy. 
Level 3: Approval and interpersonal orientation and/or stereotyped orientation-stereotyped images of good and bad persons and behaviors and considerations of others' approval and acceptance are used in justifying behaviors.

Level 4a: Self-reflective, empathic orientation-judgments include evidence of sympathetic responding, self-reflective role taking, concern with the other's humanness, and guilt or positive affect related to the consequences of one's actions.

Level 4b: Transitional level-justifications for helping or not helping involve internalized values, norms, duties, or responsibilities, or refer to the necessity of protecting the rights and dignity of other persons; these ideas, however, are not clearly and strongly stated.

Level 5: Strongly internalized stage-justifications for helping or not helping are based on internalized values, norms, or responsibilities, the desire to maintain individual and societal contractual obligations, the belief in the dignity, rights, and the equality of all individuals. Positive or negative affect related to the maintenance of self-respect for living up to one's own values and accepted norms also characterizes this stage.

The above levels are not viewed as structured wholes in the sense that a given child is expected to exhibit consistent reasoning across situations. This is because, unlike Kohlberg (1969), we code individuals' performance (all of their reasoning), not just competence (highest levels of reasoning). Thus, in this way our levels are similar to what Kohlberg has called "soft stages."

Because we wanted to examine the relation of prosocial behavior to maturity of reasoning (level) as well as to predominant type of reasoning (category) used by a child, a measure representing level of moral judgment was computed for each child (see Eisenberg et al., 1983). The level score was constructed in a manner similar to that used to score Kohlbergian prohibition-oriented reasoning; subjects were assigned composite scores by weighting the proportion of the subject's reasoning at each level (level 1 reasoning $=1$; level $2=2$; level $3=$ 3 ; level $4-5=4$; for example, $50 \%$ needs-oriented and $50 \%$ hedonistic reasoning equals 150 ). 


\section{RESULTS}

\section{AGE CHANGES IN MORAL JUDGMENT}

To examine age changes in the German children's prosocial moral judgment, 3 (age) $\times 2$ (sex) multivariate and univariate analyses of variance were computed using all reasoning categories with a mean of 4.20 (on a 4-16 scale) or higher for at least one age group (those categories defined previously). The multivariate $F$ (Pillais $F$ approximation) for the main effect of age was significant, $F(16,160)=3.64, p<.001$. Furthermore, the univariate analyses for age were at least marginally significant for hedonistic, direct reciprocity, affectional relationship, pragmatic, needs-oriented, approval/interpersonal, and reference to humanness, $F(2,86)=10.34,5.10,2.55,4.99$, 3.42 , and $6.62 ; \mathrm{p}<.001, .01, .10, .01, .05$, and .01 , respectively (see Table 1). According to Newman-Keuls tests, fourth graders used less hedonistic reasoning than preschoolers $(\mathrm{p}<$ $.01)$ and second graders $(p<.05)$. Moreover, second graders verbalized less hedonistic reasoning than did preschoolers $(\mathrm{p}<$ $.05)$. Direct reciprocity reasoning was more common among fourth graders than preschoolers $(p<.01)$; the differences between adjacent age groups were not significant. Second graders verbalized more needs-oriented reasoning than did preschoolers $(\mathrm{p}<.01)$; however, needs-oriented reasoning decreased nonsignificantly from second to fourth grade. Approval/interpersonal reasoning and concern with humanness were used more by fourth graders than preschoolers $(\mathrm{p}<.05$ and .01); for concern with humanness, the increase was also significant from second to fourth grade $(\mathrm{p}<.05)$.

To examine age differences in the prosocial composite scores, an additional 3 (age) $\times 2$ (sex) analysis of variance was computed. Only the effect for age was highly significant ( $F$ $(2,86)=19.83 ; p<.001)$. The composite score was significantly higher for second graders $(M=188)$ than for preschoolers $(M=$ $165 ; p<.01)$, and higher for fourth graders $(M=212)$ than for second graders $(p<.01)$. 
TABLE 1

Moral Reasoning Categories: Means

\begin{tabular}{|c|c|c|c|c|c|c|}
\hline \multirow[b]{2}{*}{ REASONING CATEGORIES } & \multicolumn{3}{|c|}{$\begin{array}{l}\text { GERPAAN CHILDREN } \\
\text { GRADE }\end{array}$} & \multicolumn{3}{|c|}{$\begin{array}{l}\text { AMERICAN CHILDREN } \\
\text { GRADE }\end{array}$} \\
\hline & Preschool & 2 & 4 & Preschool & 2 & 4 \\
\hline Hedonistic & 8.17 & 6.66 & 5.21 & 10.13 & 6.63 & 5.69 \\
\hline Direct reciprocity & 4.00 & 4.34 & 4.76 & 4.00 & 4.17 & 4.28 \\
\hline Affectional relationship & 4.07 & 4.24 & 4.15 & 4.00 & 4.23 & 4.36 \\
\hline Pragmat 1sm & 4.63 & 5.14 & 5.48 & 4.37 & 4.90 & 5.33 \\
\hline Needs-oriented & 10.73 & 12.90 & 12.27 & 9.67 & 12.87 & 13.18 \\
\hline Concern with humanness & 4.00 & 4.38 & 4.91 & 4.03 & 4.90 & 4.64 \\
\hline Stereotypes of good/bad persons & 4.33 & 4.38 & 4.39 & 4.70 & 5.13 & 5.67 \\
\hline Approval/1nterpersonal orientation & 4.00 & 4.03 & 4.21 & 4.00 & 4.00 & 4.26 \\
\hline Sympathet ic & 4.00 & 4.10 & 4.18 & 4.00 & 4.13 & 4.49 \\
\hline Role taking & 4.00 & 4.03 & 4.09 & 4.00 & 4.10 & 4.41 \\
\hline \multicolumn{7}{|l|}{ Positive affect/simple or } \\
\hline related to consequences & 4.00 & 4.03 & 4.00 & 4.00 & 4.00 & 4.31 \\
\hline
\end{tabular}

NOTE: The possible range for scores was 4.00 to 16.00 . Only categories with means of 4.25 for at least one age group in one country are shown.

To compare patterns of development for German and American children, 3 (age) $\times 2$ (country) $\times($ sex) multivariate and univariate analyses of variance were computed for all categories of reasoning used with any real frequency (see Table 1). None of the multivariate Fs (or even the univariate Fs) for any interactions of country with sex or age even approached marginal significance; thus, the developmental patterns were the same for German and American children. The multivariate main effect for country (and age, of course) was significant: $\mathrm{F}(11,169)=3.42, \mathrm{p}<.001$. There were significant differences for two categories. German children verbalized more direct reciprocity reasoning $(M=4.38)$ than did American children $(M=4.12)$, whereas Americans used more stereotypic reasoning $(\mathrm{M}=5.21)$ than did Germans $(\mathrm{M}=4.37) ;(\mathrm{F}(1,179)=3.99$ and $13.83 ; \mathrm{p}<.05$ and .001 , respectively). The pattern of findings was nearly identical when the data were transformed with a base 10 logarithm transformation to reduce heterogeneity of the variances. 


\section{THE RELATION OF REASONING} TO PROSOCIAL BEHAVIOR

The relations between the second and fourth-grade German children's prosocial behavior and reasoning were examined by correlating the two prosocial indices with the prosocial composite scores as well as the more frequently verbalized types of reasoning (those in the multivariate analyses for German children). Number of candies donated was positively related to needs-oriented reasoning $[\operatorname{rho}(58)=.26, \mathrm{p}<.05]$ and to the moral judgment composite $[\operatorname{rho}(58)=.31, \mathrm{p}<.02]$ and negatively related to hedonistic reasoning $[\operatorname{rho}(58)=-.43, p<$ $.001]$. Helping was unrelated to reasoning. However, because number of candies donated was positively related with age $[\mathrm{rho}(58)=.41, \mathrm{p}<.001]$, the relation between age and helping was not significant-the correlations were recomputed partialling out the effects of age. This was done by substituting Spearman rho correlations into the formula for partial correlations (Siegel, 1956). When the effects of age were controlled in this manner, donating was still positively related to needsoriented reasoning and the prosocial composite (rho $=.33$ and .22 , respectively) and negatively related to hedonistic reasoning (rho = -.35; according to Siegel, the significance level for nonparametric partial correlations cannot be computed because the sampling distribution is unknown).

\section{DISCUSSION}

In general, the results of the present study are quite similar to findings concerning prosocial development in American children. Consistent with the tenets of cognitive developmental theory (Kohlberg, 1969), the age-related developmental changes in the German children's prosocial moral judgment were very similar to those noted in prior cross-sectional and longitudinal research with American children (Eisenberg et al., 1983, 1984; Eisenberg-Berg, 1979). In fact, when the German children's reasoning was directly compared with that of American chil- 
dren, there were no differences in the pattern of age-related change. It appears that prosocial moral judgment develops similarly in different Western cultures, probably due to both the role of cognition in development and some basic similarities in the socialization of children in Western cultures.

Cross-cultural differences in prosocial reasoning regardless of age were also few. This may be due, in part, to the low rate of use of many categories in both groups (suggesting similarity in the range of categories used). Germans used more reasoning concerning direct reciprocity, whereas American children made more references to stereotypic conceptions of good and bad persons or behaviors. Perhaps the relative importance of norms relating to direct reciprocity versus norms relating to helping varies across the two countries. However, it is important to note that neither direct reciprocity nor stereotyped reasoning were frequently verbalized, especially by the preschoolers or second graders.

The relations between German children's reasoning and behavior were also reminiscent of findings for American children. Donating behavior, which has been associated with American children's needs-oriented and/or hedonistic reasoning (positively for the former and negatively for the latter type of reasoning; Eisenberg et al., 1984; Eisenberg-Berg \& Hand, 1979) was similarly correlated with German children's reasoning. It seems logical that a behavior that costs the child a possession should be associated with reasoning concerning costs to the self and benefits for the recipient. In contrast, simply helping to pick up paper clips, a low-cost behavior unlikely to evoke moral conflict, was unrelated to prosocial reasoning. This same measure (Eisenberg et al., 1984) or similar indices of helping (Eisenberg-Berg \& Hand, 1979) have been unrelated to American children's prosocial judgment. These data provide support for two important propositions: (a) that the relation between prosocial reasoning and behavior is similar in different Western countries; and (b) that this relation varies as a function of type of the prosocial action.

In summary, it appears that there are some strong similarities in the development of German and American children's 
prosocial cognitions. Moreover, the links between prosocial thought and action seem to be consistent across cultures. These findings provide some support for the cognitive developmental assumption that there are basic similarities in moral judgment across cultures (Kohlberg, 1969). Of course, a very different pattern of findings could emerge if children were studied from a culture very different from that in America. Such research is sorely needed before we draw any firm conclusions regarding the development of prosocial tendencies.

\section{REFERENCES}

Adelson, J. (1971). The political imagination of the young adolescent. Daedalus, 100, 1013-1050.

Devereux, E'. C., Bronfenbrenner, V., \& Suci, G. J. (1962). Patterns of parent behavior in the United States of America and in the Federal Republic of Germany: A cross-national comparison. International Sociological Science Journal, 14, 488506.

Eisenberg, N., Lennon, R., \& Roth, K. (1983). Prosocial development: A longitudinal study. Developmental Psychology, 19, 846-855.

Eisenberg, N., Pasternack, J. F., \& Lennon, R. (1984, March). Prosocial behavior and reasoning in middle childhood. Paper presented at the Southwest Society for Research in Human Development, Denver.

Eisenberg-Berg, N. (1979). Development of children's prosocial moral judgment. Developmental Psychology, 15, 128-137.

Eisenberg-Berg, N., \& Hand, M. (1979). The relationship of preschoolers' reasoning about prosocial moral conflicts to prosocial behavior. Child Development, 50, 356-363.

Kohlberg, L. (1969). Stage and sequence: The cognitive-developmental approach to socialization. In D. A. Goslin (Ed.), Handbook of socialization theory and research (pp. 325-480). New York: Rand-McNally.

Leahy, R. L. (1979). Development of conceptions of prosocial behavior: Information affecting rewards given for altruism and kindness. Developmental Psychology, 15, 34-37.

Lederer, G. (1982). Trends in authoritarianism: A study of adolescents in West Germany and the United States since 1945. Journal of Cross-Cultural Psychology, 13, 299-314.

O'Connor, M., Cuevas, J., \& Dollinger, S. (1981). Understanding motivations behind prosocial acts: A developmental hypothesis. Journal of Genetic Psychology, 39, 267-276.

Parikh, B. (1980). Development of moral judgment and its relation to family environment factors in Indian and American families. Child Development, 51, 1030-1039. 
Siegel, S. (1956). Nonparametric statistics. New York: McGraw-Hill.

Underwood, B., \& Moore, B. (1982). Perspective-taking and altruism. Psychological Bulletin, 91, 143-173.

Wesley, F., \& Karr, C. (1968). Vergleich der Ansichten und Erziehungshaltungen Deutscher und Amerikanischer Mutter. Psychologische Rundschau, 19, 35-46.

Nancy Eisenberg received her Ph.D. from the University of California, Berkeley, in 1976. She is currently Associate Professor at Arizona State University. She is coauthor of Roots of Caring, Sharing, and Helping: The Development of Prosocial Behavior in Children and editor of The Development of Prosocial Behavior. Her research interests include moral and sex role development.

Klaus Boehnke is a research scientist in the department of psychology at the Technische Universitaet in Berlin. His research interests include social development and substance use in adolescence.

Petra Schuhler is a research scientist at the Technische Universitaet in Berlin. Her research interests include social cognition and moral development.

Rainer K. Silbereisen is Professor in the department of psychology at the Technische Universitaet in Berlin. He is coeditor of Entwicklung des moralischen Urteilens: Theorie, Methoden, Praxis and Development as Action in Context. His research interests include social cognition, moral development and adolescents' substance use. 